\title{
A short proof for the multi- deletion error correction property of Helberg codes
}

\author{
Manabu Hagiwara ${ }^{\text {a) }}$ \\ Graduate School of Science, Chiba University, \\ 1-33 Yayoi-cho, Inage-ku, Chiba, Chiba 263-0022, Japan
}

a)hagiwara@math.s.chiba-u.ac.jp

\begin{abstract}
A short proof for the $s$-deletion error-correction property of Helberg codes is given.

Keywords: Helberg codes, deletion error, Levenshtein codes

Classification: Fundamental Theories for Communications
\end{abstract}

\section{References}

[1] V. I. Levenshtein, "Binary codes capable of correcting deletions, insertions, and reversals," Sov. Phys.-Dokl., vol. 10, no. 8, pp. 707-710, Feb. 1966.

[2] A. S. J. Helberg and H. C. Ferreira, "On multiple insertion/deletion correcting codes," IEEE Trans. Inf. Theory, vol. 48, no. 1, pp. 305-308, Jan. 2002. DOI:10.1109/18.971760

[3] K. A. S. Abdel-Ghaffar, F. Paluncic, H. C. Ferreira, and W. A. Clarke, "On Helberg's generalization of the Levenshtein code for multiple deletion/insertion error correction," IEEE Trans. Inf. Theory, vol. 58, no. 3, pp. 1804-1808, Mar. 2012. DOI:10.1109/TIT.2011.2174961

\section{Introduction}

The deletion error model is a synchronization error model over communication and storage channels and is associated with phenomena that lose a part of transmitted symbols. An $s$-deletion code $C$ is an error-correcting code that for any codeword $x \in C$ and for any set $D$ of at most $s$-indices, we can uniquely find the codeword from a deleted sequence $x^{D}$. Here $x^{D}$ is a sequence obtained from $x$ by deleting symbols with index included in $D$. Hence the length of $x^{D}$ is the length of $x$ minus the cardinality $|D|$. Another model, the insertion error model, corresponds with phenomena that receive specious symbols. Levenshtein showed $s$-deletion errorcorrecting codes, $s$-insertion error-correcting codes, and $s$-insertion/deletion (i.e., combination) error-correcting codes are all equivalent [1]. Note that a substitution error, e.g., 0 is flipped to 1 , is a combination of insertion and deletion errors, e.g., 0 is changed to 01 and then subsequently changed to 1 . Therefore multi deletion error correcting code is regarded as more general than a multi bit-flip error-correcting code which is often discussed for communication theory. 
Helberg codes $C^{s, n}(a)$ of length $n$ and of parameter $a$ [2] are a class of $s$-deletion error correction codes and are known as a generalization of Levenshtein codes [1]. The code space $C^{s, n}(a)$ is defined as

$$
C^{s, n}(a):=\left\{x \in\{0,1\}^{n} \mid\left\langle x, \lambda^{(s)}\right\rangle \equiv a\left(\bmod \lambda_{n+1}^{(s)}\right)\right\},
$$

where $x=\left(x_{1}, x_{2}, \ldots, x_{n}\right), \lambda^{(s)}=\left(\lambda_{1}^{(s)}, \lambda_{2}^{(s)}, \ldots, \lambda_{n}^{(s)}\right)$, and $\langle$,$\rangle is a standard inner$ product. In the definition of $C^{s, n}(a),\left\{\lambda_{i}^{(s)}\right\}$ is an integer sequence defined as $\lambda_{i}^{(s)}:=0$ for $i \leq 0$ and $\lambda_{i}^{(s)}:=1+\sum_{1 \leq j \leq s} \lambda_{i-j}^{(s)}$. Therefore, for $i>0$, we have positivity, i.e., $\lambda_{i}^{(s)}>0$ and the strictly increasing property $\lambda_{i}^{(s)}<\lambda_{i+1}^{(s)}$. Note that $C^{s, n}(a)$ is an integer sequence but not a binary field one.

Abdel-Ghaffar et al. first proved that Helberg codes were $s$-deletion errorcorrecting codes [3]. To prove that, they showed two following key lemmas:

Lemma 1.1 (Lemma 2 in [3]). For any binary sequence $x, y$ of length $n$ and any index set $D, D^{\prime} \subset\{1,2, \ldots, n\}$, if $|D|,\left|D^{\prime}\right| \leq s$ and $x^{D}=y^{D^{\prime}}$, we obtain

$$
-\lambda_{n+1}^{(s)}<\left\langle x, \lambda^{(s)}\right\rangle-\left\langle y, \lambda^{(s)}\right\rangle<\lambda_{n+1}^{(s)} .
$$

Lemma 1.2 (Lemma 3 in [3]). For any codeword $x, y \in C^{s, n}(a)$, and any index set $D, D^{\prime} \subset\{1,2, \ldots, n\}$, if $|D|,\left|D^{\prime}\right| \leq s$ and $x^{D}=y^{D^{\prime}}$, we obtain

$$
\left\langle x, \lambda^{(s)}\right\rangle \neq\left\langle y, \lambda^{(s)}\right\rangle \text {. }
$$

By combining the lemmas above, it directly follows that Helberg codes are $s$-deletion error-correcting codes.

This letter gives a short proof of the lemmas above based on the simple observations: a) for any $x \in\{0,1\}^{n}$ let $x^{\prime}$ be a sequence obtained by replacing some entries of $x$ with 0 (resp. with 1). This implies $\left\langle x, \lambda^{(s)}\right\rangle \geq\left\langle x^{\prime}, \lambda^{(s)}\right\rangle$ (resp. $\left.\left\langle x, \lambda^{(s)}\right\rangle \leq\left\langle x^{\prime}, \lambda^{(s)}\right\rangle\right)$. b) for any $x \in\{0,1\}^{n}$ and a sequence $x^{\prime}$ obtained by permuting the $i$ th element $x_{i}$ and the $(i+1)$ th element of $x$, if $x_{i}=0$ (resp. $x_{i}=1$ ), we have $\left\langle x, \lambda^{(s)}\right\rangle \geq\left\langle x^{\prime}, \lambda^{(s)}\right\rangle$ (resp. $\left.\left\langle x, \lambda^{(s)}\right\rangle \leq\left\langle x^{\prime}, \lambda^{(s)}\right\rangle\right)$.

Repeatedly using observations a) and b) implies

$$
\left\langle x^{D} 0^{|D|}, \lambda^{(s)}\right\rangle \leq\left\langle x, \lambda^{(s)}\right\rangle \leq\left\langle x^{D} 1^{|D|}, \lambda^{(s)}\right\rangle,
$$

where $x^{D} 0^{|D|}\left(\right.$ resp. $\left.x^{D} 1^{|D|}\right)$ is a concatenated sequence of $x^{D}$ and all 0 (resp. all 1) sequence of length $|D|$.

New Proof of Lemma 1.1. $\left\langle x, \lambda^{(s)}\right\rangle-\left\langle y, \lambda^{(s)}\right\rangle \leq\left\langle x^{D} 1^{|D|}, \lambda^{(s)}\right\rangle-\left\langle y^{D^{\prime}} 0^{\left|D^{\prime}\right|}, \lambda^{(s)}\right\rangle=$ $\left\langle 0^{n-|D|} 1^{|D|}, \lambda^{(s)}\right\rangle \leq \sum_{1 \leq j \leq n} \lambda_{n+1-j}^{(s)}<\lambda_{n+1}^{(s)}$. The 1st inequality is from (1). The next equality is from the assumption $x^{D}=y^{D^{\prime}}$. The next inequality is from the assumption $|D| \leq s$. The last is from the definition of $\lambda^{(s)}$.

New Proof of Lemma 1.2. For $1 \leq i \leq n$, let $x_{\mid i}$ denote a subsequence of $x$ which consists of the first $i$ entries. By the assumption $x \neq y$, we can assume $x=x_{\mid I} 1 \mathrm{w}$ and $y=y_{\mid I} 0 w$ for some $w \in\{0,1\}^{n-I-1}$ and some $1 \leq I<n$, without loss of generality. It follows that $\left\langle x, \lambda^{(s)}\right\rangle-\left\langle y, \lambda^{(s)}\right\rangle=\lambda_{I+1}^{(s)}+\left\langle x_{\mid I}, \lambda_{\mid I}^{(s)}\right\rangle-\left\langle y_{\mid I}, \lambda_{\mid I}^{(s)}\right\rangle$.

On the other hand, we have $\left\langle x_{\mid I}, \lambda_{\mid I}^{(s)}\right\rangle-\left\langle y_{\mid I}, \lambda_{\mid I}^{(s)}\right\rangle \geq\left\langle z_{\mid I-e} 0^{e}, \lambda_{\mid I}^{(s)}\right\rangle-$ $\left\langle z_{\mid I-f} 1^{f}, \lambda_{\mid I}^{(s)}\right\rangle \geq\left\langle z_{\mid I-s} 0^{s}, \lambda_{\mid I}^{(s)}\right\rangle-\left\langle z_{\mid I-s} 1^{s}, \lambda_{\mid I}^{(s)}\right\rangle$, where $z=x^{D}\left(=y^{D^{\prime}}\right), \quad e=\mid\{1 \leq$ $i \leq I \mid i \in D\} \mid$ and $f=\left|\left\{1 \leq i \leq I \mid i \in D^{\prime}\right\}\right|$. The first inequality is from (1) and the second one is from the assumption $|D|,\left|D^{\prime}\right| \leq s$. 
Furthermore we have $\left\langle z_{\mid I-s} 0^{s}, \lambda_{\mid I}^{(s)}\right\rangle-\left\langle z_{\mid I-s} 1^{s}, \lambda_{\mid I}^{(s)}\right\rangle=-\left\langle 0^{I-s} 1^{s}, \lambda_{\mid I}^{(s)}\right\rangle=$ $-\sum_{i=1}^{s} \lambda_{I+1-i}^{(s)}>-\lambda_{I+1}^{(s)}$. The last inequality is from the definition of $\lambda_{I+1}^{(s)}$. It follows that $\left\langle x, \lambda^{(s)}\right\rangle>\left\langle y, \lambda^{(s)}\right\rangle$.

\section{Acknowledgments}

This work is partially supported by KAKENHI (B) 25289118 and 26289116 . 